sen Korrelation mit körperlicher Fitness. Verhaltenscharakteristika unmittelbar vor dem Ereignis, die das Takedownrisiko erhöhten, waren unklare Gedanken und Lautstärke des verbalen Ausdrucks.

\section{Warum sind japanische Patienten zufriedener?}

„Psychiatrische Pflegekräfte empfinden Zwangsmaßnahmen in der Regel als notwendiges Übel“, weiß Ada Hui, Universität Nottingham, UK, aus einer eigenen Untersuchung. Nicht wenige scheinen aber auch erhebliche Zweifel am Sinn von Isolierung und Fixierung zu haben. Pãivi Soininen, Kellokoski Hospital, Tuusula, Finnland, präsentierte eine Studie, die sich mit der Frage beschäftigte, wie Patienten psychiatrischer Akutkliniken in Finnland und Japan Zwangsmaßnahmen erleben. Ein erstaunliches Ergebnis der Studie war, dass japanische Patienten mit der Behandlung zufriedener waren als finnische, obwohl in japanischen Kliniken ein ungünstigerer Personalschlüssel herrscht und sie durchschnittlich fast dreimal so lange fixiert oder isoliert waren. Soininen vermutet, dass sich hier kulturelle Unterschiede zeigen, die sich auch auf die Grundhaltung psychiatrischen Personals auswirken: „In Japan ist man mehr darum bemüht, eine kooperative Beziehung aufzubauen; in Finnland dominiert dagegen das Streben nach Sicherheit und Schutz im Namen des Gesetzes“. In Japan sei Harmonie der fundamentale Wert; die Kardinaltugenden der Krankenpflege seien Höflichkeit und Respekt für andere Menschen.

\section{Mehr sprechen - mit Patienten und Mitarbeitern}

Sowohl finnische als auch japanische Patienten fanden den Gebrauch von Isolierung oder Fixierung unnötig und wünschten sich, dass ihre Meinung nicht nur gehört, sondern auch umgesetzt wird. Dafür wäre nach Soininens Einschätzung ein echter Dialog zwischen Patienten und Personal notwendig und - statt Routine - das Ausloten von Alternativen im Rahmen einer individualisierten Versorgung.
„Sprechen Sie mehr und offener mit Ihren Patienten“ lautet der dringliche Rat der psychiatrieinternen Konfliktforscher. Das heißt beispielsweise, den Patienten soweit wie möglich in die Behandlungsplanung mit einzubeziehen, wenn man Zwangmaßnahmen in Erwägung zieht, aber auch - im Rahmen der Möglichkeiten - während und unbedingt nach erfolgtem Einsatz von körperlichem Zwang, Fixierung oder Isolierung. Ein weiterer Rat lautet, regelmäßig im Team anhand von konkreten Vorfällen über Möglichkeiten nachzudenken, wie man zukünftig anders reagieren und früher deeskalieren (siehe Infobox) könnte.

Thomas M. Heim, freier Medizinjournalist

Symposium „The use of coercive measures in forensic psychiatry", 21. EPA, Nizza, 7.4.2013

\section{Deeskalierende Maßnahmen (Beispiele)}

- Verbale Deeskalation (Zuhören, Interesse an und Verständnis für die Situation des Patienten signalisieren, „talking down“)

- Anbieten von Gesprächen mit anderen (Ober-, Chefarzt, Geistlicher, Patientenfürsprecher, Freunde und Verwandte, andere Vertrauenspersonen)

- Verbale Grenzsetzung (bestimmte, eindeutige Benennung von Verhalten, welches unerwünscht und nicht tolerabel ist)

- Anbieten eines Getränks, von Essen oder einer Süßigkeit, einer Zigarette, gemeinsam einen Kaffee trinken, eine Zigarette rauchen

- Anbieten von Medikamenten

- Anbieten eines "time out“ im eigenen Zimmer, im Garten, in der Klinikkapelle oder ähnlichem

- Anbieten eines warmen Bads

- Einbinden des Patienten in eine Tätigkeit auf Station, im Garten

- Anbieten von Bewegung (Tischtennis, Laufen oder ähnliches)

Quelle: Auert et al. Leitlinien für den Umgang mit der Erstbegegnung eines Patienten mit Psychiatrischen Kliniken/Fachabteilungen. http://www.lvpe-rlp.de/kategorie/leitlinie

\title{
Soziale Kontakte verringern Stigmatisierung
}

\author{
Was fast jeder schon im Alltag beobachten konnte, \\ bestätigt nun die Anti-Stigma-Studie: Persönliche \\ soziale Kontakte verringern Stigmatisierung und \\ Diskriminierung.
}

Stigmata gegenüber Menschen mit psychischen Krankheiten wie Schizophrenie und Depression sind in der Bevölkerung einschließlich des medizinischen Personals - nach wie vor weit verbreitet, berichtete Dr. Sara Evans-Lacko, London. Die Ergebnisse verschiedener Studien deuten darauf hin, dass die Allgemeinbevölkerung weniger zu Stigmatisierung und Diskriminierung von Menschen mit psychischen Erkrankungen neigt, wenn sie den Betroffenen begegnet sind. Je intensiver der soziale Kontakt, desto ausgeprägter sei die erzielte Veränderung der Einstellung in der Bevölkerung.

Im Rahmen der britischen Anti-Stigma-Kampagne „Time to Change" untersuchten Evans-Lacko und Kollegen die Wirksam- keit einer solchen Aufklärungs- und Kontaktinitiative. Ziel der Initiative war es, Bewusstsein und Wissen über psychische Krankheiten und Therapiemöglichkeiten zu verbessern, öffentliche Einstellungen gegenüber den Betroffenen zu verändern und über die Vermittlung direkter Kontakte mit psychisch Kranken oder indirekter Kontakte (mittels DVDs) die Diskriminierung und Vorurteile der Bevölkerung zu verringern. Die Anti-Stigma-Studie bestätigte, dass sozialer Kontakt prinzipiell geeignet ist, um Vorurteile und Diskriminierung zu reduzieren, Verhaltensveränderungen zu erreichen und Empathie aufzubauen, vor allem in Kombination mit edukativen Maßnahmen. Allerdings scheint der Erfolg dieser Maßnahmen nur vorübergehende Wirkung zu haben, räumte Evans-Lacko ein. Daher sei es wichtig, Anti-Stigma-Kampagnen langfristig anzulegen.

Abdol A. Ameri, freier Medizinjournalist

Symposium „I don't to talk about it - The role of stigma suicide prevention", 21. EPA, Nizza, 6. - 9.4.2013 